\title{
Objectified Body Consciousness in Relation to Recovery from an Eating Disorder
}

\author{
Ellen E. Fitzsimmons ${ }^{a}$, Anna M. Bardone-Cone ${ }^{a}$, and Kathleen A. Kelly ${ }^{a}$ \\ aUniversity of North Carolina at Chapel Hill, Department of Psychology, CB\#3270-Davie Hall, \\ Chapel Hill, NC, 27599, United States
}

\begin{abstract}
In Western society, the feminine body has been positioned as an object to be looked at and sexually gazed upon; thus, females often learn to view themselves as objects to be observed (i.e., objectified body consciousness (OBC)). This study examined the relation between $\mathrm{OBC}$ and eating disorder recovery by comparing its components across non-eating disorder controls, fully recovered, partially recovered, and active eating disorder cases. Results revealed that non-eating disorder controls and fully recovered individuals had similarly low levels of two components of $\mathrm{OBC}$, body surveillance and body shame. Partially recovered individuals looked more similar to those with an active eating disorder on these constructs. The third component of OBC, control beliefs, and a conceptually similar construct, weight/shape self-efficacy, did not differ across groups. Results provide support for the importance of measuring aspects of self-objectification, particularly body surveillance and body shame, across the course of an eating disorder.
\end{abstract}

\section{Keywords}

self-objectification; objectified body consciousness; eating disorders; recovery

\section{Introduction}

Objectification theory holds that in Western society, women internalize the societal message that their bodies are objects, valuable as something to be gazed upon or used by others, and learn to view themselves as objects to be looked at (Fredrickson \& Roberts, 1997). A woman in American society may feel as if her "sexual parts or functions are separated out

\footnotetext{
(C) 2011 Elsevier Ltd. All rights reserved.

Correspondence concerning this article should be addressed to Anna M. Bardone-Cone, Tel.: +1 9199625989 ; fax: +1 9199622537 . ellen.fitzsimmons@unc.edu (E.E. Fitzsimmons), bardonecone@unc.edu (A.M. Bardone-Cone), kakelly@email.unc.edu (K.A. Kelly) The authors note that findings regarding the Body Shame subscale of the Objectified Body Consciousness Scale were previously published in Bardone-Cone et al. (2010) as a way to validate a new definition of eating disorder recovery; however, the authors believed it to be important to report the body shame findings again here in an effort to provide the reader with a more complete picture of what the components of objectified body consciousness look like in relation to recovery from an eating disorder. This is the first piece of work to focus on the full set of constructs related to objectified body consciousness in relation to eating disorder recovery.

Publisher's Disclaimer: This is a PDF file of an unedited manuscript that has been accepted for publication. As a service to our customers we are providing this early version of the manuscript. The manuscript will undergo copyediting, typesetting, and review of the resulting proof before it is published in its final citable form. Please note that during the production process errors may be discovered which could affect the content, and all legal disclaimers that apply to the journal pertain.

Contributors Anna Bardone-Cone designed the study and wrote the protocol. Ellen Fitzsimmons conducted the literature searches and statistical analyses and wrote the first draft of the manuscript. Kathleen Kelly conducted additional literature searches and contributed to the first draft of the manuscript. Ellen Fitzsimmons, Anna Bardone-Cone, and Kathleen Kelly contributed to draft revisions and have approved the final manuscript.
}

Conflict of Interest All authors declare that they have no conflicts of interest. 
from her, reduced to the status of mere instruments, or else regarded as if they were capable of representing her" (Bartky, 1990, p. 35). Further, this message communicates that there is a certain form that a woman's body should take as object: thin (McKinley \& Hyde, 1996). This adoption of the outsider's perspective of one's own body is known as objectified body consciousness (OBC), which consists of three components: body surveillance, body shame, and control beliefs (McKinley \& Hyde, 1996), and has been associated with a number of negative consequences including depression, anxiety, decreased well-being, body dissatisfaction, and disordered eating (Fredrickson \& Roberts, 1997; McKinley \& Hyde, 1996; Mercurio \& Landry, 2008; Miner-Rubino, Twenge, \& Fredrickson, 2002; Noll \& Fredrickson, 1998; Tylka \& Hill, 2004).

Because OBC can negatively impact body image (e.g., Harper \& Tiggemann, 2008) and lead to eating disorder symptomatology (e.g., Noll \& Fredrickson, 1998), elevated levels of selfobjectification among those in the process of recovering from an eating disorder could worsen prognosis or increase risk for relapse. Relatedly, it may be that attaining levels of self-objectification similar to those of controls with no history of an eating disorder is essential to reaching full eating disorder recovery. The current study seeks to understand how the various components of OBC (body surveillance, body shame, and control beliefs) compare across different stages of an eating disorder.

The body surveillance component of OBC involves continual self-monitoring of body shape and weight in order to ensure conformity to culturally and socially imposed standards of beauty (McKinley \& Hyde, 1996). This self-monitoring emerges from having internalized cultural standards of the ideal body and the observer's perspective of oneself as an object to be gazed upon (Fredrickson \& Roberts, 1997). Indeed, research has indicated that body surveillance is the behavioral manifestation of self-objectification; that is, if a woman has internalized the observer's perspective of her own body, she will engage in persistent body surveillance or monitoring (e.g., Moradi, 2010, 2011). While it theoretically could be implemented in a neutral way as a means to check whether one is attaining an appearance standard, the reality is that most women will come to recognize that their bodies are far from the unattainable cultural body standard, and as a result, may experience body dissatisfaction and adopt unhealthy or disordered eating patterns (McKinley \& Hyde, 1996). As previously suggested, body surveillance may also contribute to the maintenance of an eating disorder or increase risk for relapse among individuals recovered from an eating disorder.

The impossibly thin cultural ideal that many women in Western society strive for creates a discrepancy between the perception of the real self and the desired self, which can produce body shame, the affective component of OBC (Harrison, 2001; McKinley \& Hyde, 1996). As conceptualized by objectification researchers, body shame stems from a sense of failure and wrongdoing, and it extends beyond just negative attitudes about one's body to negative attitudes about oneself (McKinley \& Hyde, 1996; Noll \& Fredrickson, 1998). Further, this shame stems from a sense of "exposure" (e.g., being exposed as one who does not live up to cultural standards) and is typically coupled with feelings of inferiority and defectiveness (McFarland \& Baker-Baumann, 1990). The more women self-objectify and internalize the thin ideal, the more likely they are to experience body shame and negative affect (Myers \& Crowther, 2007), which may in turn lead to unhealthy eating patterns (Fredrickson \& Roberts, 1997).

The third component of $\mathrm{OBC}$, control beliefs, refers to the belief that one has the ability to control one's body and appearance through one's actions and that effort can override genetic influences on weight/shape (McKinley \& Hyde, 1996). In contrast to body surveillance and body shame, this aspect of $\mathrm{OBC}$ is not as clearly related to disordered eating. While some studies have found that women who believe they can and should control their weight are 
more likely to have higher rates of body dissatisfaction and eating disorder symptomatology (e.g., Furnham \& Atkins, 1997; Laliberte, Newton, McCabe, \& Mills, 2007; McKinley, 1998), other research has found no connection between control beliefs and eating disorder symptomatology (e.g., Basow, Foran, \& Bookwala, 2007; McKinley, 1999; Tylka, 2004). Due to the inconsistent, but largely null, findings regarding the relation between control beliefs and eating disorder symptoms, we also examined another construct that is conceptually similar to control beliefs: weight/shape self-efficacy, which assesses confidence related to being able to attain one's desired weight/shape.

Research has shown that both body surveillance (the indicator of self-objectification) and body shame are strong correlates of eating disordered behavior (e.g., Calogero, Davis, \& Thompson, 2005; Greenleaf, 2005; McKinley, 1999; Muehlenkamp \& Saris-Baglama, 2002; Noll \& Fredrickson, 1998; Striegel-Moore \& Bulik, 2007; Tiggemann \& Slater, 2001). In the one known study that has investigated self-objectification among women with diagnosable eating disorders, results indicated that self-objectification partially mediated the relation between thin ideal internalization and drive for thinness and that body shame partially mediated the relation between self-objectification and drive for thinness (Calogero et al., 2005). However, what is not yet known is what these constructs related to OBC (as well as control beliefs and weight/shape self-efficacy) look like as an individual recovers from an eating disorder. Because OBC can negatively impact body image (e.g., Harper \& Tiggemann, 2008) and because it is associated with eating disorder symptomatology (e.g., Noll \& Fredrickson, 1998), it is important to understand exactly what these constructs look like as individuals recover from an eating disorder. Further, as noted by Calogero and colleagues (2005), "when women continue to view themselves from a third person, rather than a first person, perspective, factors that contribute significantly to eating disorders pathology remain untouched" (p. 48). That is, unless this socially constructed perception of the self is challenged, progress in other areas (e.g., eating patterns, family dynamics) may be difficult, as well. The present study answers the call of Calogero et al. (2005) for selfobjectification to be examined in relation to recovery from an eating disorder.

As suggested by Calogero and colleagues (2005), it may be that high levels of selfobjectification help maintain individuals in an incomplete state of recovery because they still perceive themselves in this socially constructed manner and that full recovery is more closely linked with obtaining levels of body surveillance and body shame (and potentially control beliefs and weight/shape self-efficacy) similar to those of non-eating disorder controls. Thus, in this study, we chose to examine the aspects of OBC across stages of eating disorder recovery, with particular interest in the self-reported levels of these constructs among those with evidence for being fully recovered according to physical, behavioral, and psychological indices (Bardone-Cone et al., 2010). Those fully recovered were compared to individuals currently diagnosed with an eating disorder, individuals partially recovered (physically and behaviorally recovered, but not psychologically recovered - e.g., still thinking a great deal about food), and a non-eating disorder control group. We hypothesized that individuals who were fully recovered would report less body surveillance and body shame than either those who were partially recovered or those who met criteria for an active eating disorder, and that their levels of these constructs would be comparable to non-eating disorder controls. Given the largely null findings regarding the relation between control beliefs and eating disorder symptomatology, we hypothesized that neither control beliefs nor weight/shape self-efficacy would differ across the groups. To our knowledge, this is the first study to comprehensively focus on examining $\mathrm{OBC}$ as it relates to recovery from an eating disorder. 


\section{Method}

\subsection{Participants and Recruitment}

Attempts were made to contact all female eating disorder patients (ages 16 and older) seen at the University of Missouri Pediatric and Adolescent Specialty Clinic $(N=273)$ between 1996 and 2007. This clinic is a primary care and referral clinic specializing in the care of children and adolescents (ages 10-25 years) that has physicians with expertise in eating disorders. Patients identified as having an eating disorder were sent a mailing to their address on file (or to updated addresses found on public registries or via paid tracking services) describing the study and inviting their participation by calling the second author. If no response was received, a second mailing went out, followed by several attempts by the second author to reach the patients by phone.

Of the 273 eating disorder patients identified, 96 (35.2\%) were successfully contacted and recruited. Fifty-five $(20.1 \%)$ of the 273 were contacted but did not participate due to other time commitments or lack of interest. Of the remaining patients, four $(1.5 \%)$ were deceased and 118 patients (43.2\%) could not be contacted due to absent or incorrect mailing addresses or inability to make phone contact. These rates are fairly comparable to those of other studies doing a first follow-up of eating disorder patients over a range of about 10 years (Reas, Williamson, Martin, \& Zucker, 2000; Yackobovitch-Gavan et al., 2009), and results indicated that participants were not significantly different from non-participants on clinical variables such as eating disorder diagnoses, age, or BMI at first clinic visit. In sum, of the 151 eating disorder patients we were able to contact, 96 (64\%) participated. Non-eating disorder controls were recruited from two sources: the clinic from which the eating disorder patients were recruited $(n=17)$ and introductory psychology courses on the university campus $(n=50)$ (i.e., total number of control participants $=67)$. Of note, the controls recruited from the clinic simply had a primary care physician at the clinic and would visit the clinic for minor medical problems (e.g., the flu). All eligible controls were females ages 16 and older with no current or past eating disorder symptoms.

Participants ranged in age from 16 to 40 years $(M=21.78$ years, $S D=4.28)$, with most (91.6\%) identifying as Caucasian, $1.3 \%$ as African American, $1.9 \%$ as Asian, and $5.0 \%$ as biracial/biethnic. In terms of socio-economic status, participants' highest level of parental education ranged from 11 to 21 years $(M=16.60$ years, $S D=2.73$, with 16 years of education corresponding to having graduated from a 4-year college). The groups were similar in terms of ethnicity and socio-economic status, but differed in age $(F(3,151)=$ $15.44, p<.001$ ), with non-eating disorder controls significantly younger than the eating disorder groups (non-eating disorder controls: $M=19.46, S D=1.88$; fully recovered: $M=$ 24.55, $S D=4.89$; partially recovered: $M=23.53, S D=5.80$; active eating disorder: $M=$ $23.18, S D=4.39$ ). Because of the wide range of ages of the study participants (16 to 40 years) and the group differences in age, results with age covaried are reported, although including age as a covariate or not resulted in the same pattern of significance.

\subsection{Study Procedures}

After providing written consent (with minors providing assent and parental consent), all participants first completed the same set of questionnaires and then, on a separate date, an interview that included a diagnostic clinical interview. For the majority of the participants, the time between questionnaires and interview was within one week. Most participants completed the questionnaires $(71.2 \%)$ and interview (82.9\%) in person. Those who lived too far away to travel to the study site completed the questionnaires via mail and did a phone interview. Interviews were privately conducted by one of three extensively trained individuals (i.e., a PhD-level clinical psychologist, an advanced graduate student, and an 
advanced undergraduate student; all female) who participated in over 50 hours of training videos, role plays, and discussions about interviewing. Participants were provided financial remuneration (or, in the case of the introductory psychology students, course credit) after completing the interview. Current and former eating disorder patients received $\$ 50$ and noneating disorder controls recruited from outside psychology classes received $\$ 35$, with the difference reflecting differences in time in the study (e.g., longer interviews due to greater diagnostic complexity). All aspects of this study were approved by the university's institutional review board.

\subsection{Measures}

\subsubsection{Body Surveillance subscale of the Objectified Body Consciousness} Scale (OBCS; McKinley \& Hyde, 1996)—Body surveillance, or "viewing the body as an outside observer" (p. 181), was measured with the Body Surveillance subscale of the OBCS (McKinley \& Hyde, 1996). This subscale consists of eight items that are rated on a 7point scale ranging from strongly disagree (1) to strongly agree (7), with higher scores indicating higher levels of surveillance or habitual body monitoring and thinking of one's body in terms of how it looks rather than how one feels. McKinley and Hyde (1996) reported a coefficient alpha of 89 in a sample of student and nonstudent women, and in the current study, alpha was .87. Construct validity is demonstrated by high correlations with public self-consciousness $(r=.73)$ and nonsignificant relations with private selfconsciousness (McKinley \& Hyde, 1996).

2.3.2. Body Shame subscale of the OBCS (McKinley \& Hyde, 1996)—The Body Shame subscale of the OBCS (McKinley \& Hyde, 1996) is an 8-item subscale that quantifies "feeling shame when the body does not conform [to cultural standards]" (p. 181). Items are rated on a 7-point scale ranging from strongly disagree (1) to strongly agree (7), with higher scores indicating higher levels of body shame and feelings of being a bad person if one does not fulfill cultural expectations of one's body. McKinley and Hyde (1996) reported a coefficient alpha of .75; in the current sample, alpha was .90. Construct validity is demonstrated by the subscale's strong negative correlation with body esteem $(r=-.51$; McKinley \& Hyde, 1996).

\subsubsection{Control Beliefs subscale of the OBCS (McKinley \& Hyde, 1996)-The} Control Beliefs subscale of the OBCS (McKinley \& Hyde, 1996) is an 8-item subscale that quantifies the belief that one can control her weight and appearance if she tries hard enough. Items are rated on a 7-point scale ranging from strongly disagree (1) to strongly agree (7), with higher scores indicating that the belief that the body can be controlled with enough effort is strongly held. This contrasts with the low scorer who believes she does not control her weight or appearance and that these are controlled by factors such as heredity. McKinley and Hyde (1996) reported a coefficient alpha of .72; in this study, alpha was .74. Construct validity is demonstrated by the fact that restrained eaters believed more strongly that they could control how their body looks than women in a nonrestrained eating group (McKinley $\&$ Hyde, 1996). Discriminant validity is reflected by control beliefs being significantly positively correlated with dieting $(r=.38)$ and unrelated to shaving, using nail polish, or perming or dying hair (McKinley \& Hyde, 1996).

For all OBCS subscales, if more than two items are missing for any subscale (with a "not applicable" option being counted as missing), then the score for that subscale is not computed (McKinley \& Hyde, 1996). Fifteen study participants had more than two items missing on the Body Shame subscale and were thus excluded from analyses involving this subscale. No participant had more than two items missing for either of the other OBCS subscales. 
2.3.4. Weight/Shape Self-Efficacy (Bardone-Cone, 2002)—Weight/shape selfefficacy (17 items) was assessed using a modified version of the general subscale of the Self-Efficacy Scale developed by Sherer et al. (1982). The phrasing of the original items was modified to reflect self-efficacy related to weight/shape (i.e., confidence related to being able to attain one's desired weight/shape; Bardone-Cone, 2002). Items are rated on a 5-point scale ranging from disagree (1) to agree (5), with higher scores indicating higher levels of efficacy related to achieving the weight/shape one wants. The general self-efficacy subscale has demonstrated good reliability, with an alpha of .86 (Sherer et al., 1982), as well as good construct validity and criterion validity (Sherer \& Adams, 1983; Sherer et al., 1982).

Validity of the weight/shape self-efficacy measure is evidenced by the scale's modest correlation with general self-efficacy $(r=.39)$ (Bardone-Cone, 2002); that is, weight/shape self-efficacy is clearly related to but also distinct from general self-efficacy. Prior use of this weight/shape self-efficacy scale yielded coefficient alphas of .93 (Cain, Bardone-Cone, Abramson, Vohs, \& Joiner, 2010) and .89 and .92 (Bardone-Cone \& Cass, 2007). In the current study, alpha for the weight/shape self-efficacy scale was .89 .

2.3.5. Measures Used to Define Eating Disorder Status-To determine whether criteria for a current eating disorder were met, we used the Structured Clinical Interview for DSM-IV, Patient Edition (SCID; First, Spitzer, Gibbon, \& Williams, 1995) and determined diagnoses of anorexia nervosa (AN) - excluding the amenhorrea requirement, bulimia nervosa (BN), and Eating Disorder Not Otherwise Specified (EDNOS). A number of studies have used the SCID as the "gold standard" in terms of determining the accuracy of clinical diagnoses (Shear et al., 2000; Steiner, Tebes, Sledge \& Walker, 1995) and exhibiting strong inter-rater reliability (e.g., .77 for any eating disorder; Zanarini et al., 2000). In the current study, a random subset (approximately 5\%) of the audiotaped interviews was examined for inter-rater reliability, yielding absolute agreement between the second author and the two other interviewers for current AN, BN, and EDNOS. To determine physical recovery, we measured weight and height after the interview to determine body mass index (BMI); for the minority who did not complete the interview in person, we used self-reported weight and height to compute BMI. Of note, Smith, Hohlstein, and Atlas (1992) found that women with eating disorder symptomatology are neither more nor less accurate at reporting weight than control women. To determine behavioral recovery (i.e., no binge eating, purging, or fasting in the past three months), we used portions of the Eating Disorders Longitudinal Interval Follow-up Evaluation Interview (LIFE EAT II; Herzog et al., 1993) (a semi-structured interview) to ask, week by week, about these behaviors over the past three months. To determine psychological recovery, we used the Eating Disorder Examination-Questionnaire (EDE-Q; Fairburn \& Beglin, 1994). The EDE-Q is a self-report measure with 36 items that assesses disordered eating thoughts and behaviors over the past four weeks, yielding four subscales: Restraint (attempts to restrict food intake), Eating Concern (feeling guilty and concerned about eating), Weight Concern (dissatisfaction with and overvaluation of weight), and Shape Concern (dissatisfaction with and overvaluation of shape). In the current study, coefficient alphas were $\geq .85$ for all subscales. The SCID, LIFE EAT II, and EDE-Q are all well-established measures with good psychometric properties (Anderson \& Williamson, 2002; Fairburn \& Beglin, 1994; Herzog et al., 1993; Luce \& Crowther, 1999; Segal, Hersen, \& Van Hasselt, 1994).

\subsection{Defining Eating Disorder Recovery}

Based on data from the SCID, the LIFE EAT II, the EDE-Q, and BMI, participants were categorized into one of four groups: non-eating disorder controls, fully recovered eating disorder, partially recovered eating disorder, or active eating disorder. Non-eating disorder controls $(n=67)$ had no history of an eating disorder, and active eating disorder cases $(n=$ 53) had a current eating disorder diagnosis (AN, BN, or EDNOS). Of the active eating 
disorder group, $17 \%$ currently had AN, 6\% had BN, and 77\% had EDNOS, most with bulimic-type presentations. As recommended by Couturier and Lock (2006) and as illustrated in Bardone-Cone et al. (2010), we combined physical, behavioral, and psychological indices to assess recovery. The fully recovered group $(n=20)$ comprised women without a current eating disorder who had a BMI of at least $18.5 \mathrm{~kg} / \mathrm{m}^{2}$, which is the lower end of "normal" per the World Health Organization (Bjorntorp, 2002), reported no binge eating, purging, or fasting in the prior three months, and scored within $1 S D$ of agematched community norms on each of the EDE-Q subscales (Mond, Hay, Roders, \& Owen, 2006). This operationalization of full recovery has been validated using a wide range of measures of disordered eating attitudes and behaviors (Bardone-Cone et al., 2010). Individuals were considered partially recovered $(n=15)$ if they met all the criteria of full recovery except for psychological recovery (i.e., physical and behavioral recovery but at least one EDE-Q subscale greater than $1 S D$ of age-matched norms). Of note, eight of the study participants did not meet criteria for a current eating disorder or either definition of recovery (i.e., partial or full) and were excluded from analyses (these eight individuals are not included in the $n$ 's listed above). These were primarily individuals who had reported some (though minimal) binge eating or purging, typically once or twice in the past three months. When analyses were run including these eight individuals in the "partial recovery" group, the same pattern of results emerged as presented in the current work (using the stricter definition of partial recovery).

\section{Results}

Body surveillance and body shame were positively correlated, as expected $(r=.70, p<.001)$. Further, control beliefs were unrelated to both body surveillance $(r=.13, p=.119)$ and body shame $(r=.00, p=.969)$; similarly, weight/shape self-efficacy was unrelated to both body surveillance $(r=-.15, p=.064)$ and body shame $(r=-.16, p=.068)$, as well. As expected, weight/shape self-efficacy was positively correlated with control beliefs $(r=.29, p<.001)$, albeit to a lesser degree than was expected based on the conceptual similarities between these two constructs.

Because we wished to test for differences across the groups on all of these inter-related study variables while controlling for age, multivariate analysis of covariance (MANCOVA) was used with the three OBCS subscales and the weight/shape self-efficacy scale as a set of dependent variables and age as a covariate. The MANCOVA revealed that after controlling for age, OBCS subscales and weight/shape self-efficacy varied across groups, $F(12,350)=$ 9.64, Wilks' Lambda $=.47, p<.001$, partial $\eta^{2}=.22$. In order to further examine the nature of these group differences, univariate tests controlling for age were conducted (see Table 1). Fully recovered individuals and non-eating disorder controls had very similar levels of both body surveillance and body shame that were significantly lower than partially recovered individuals and those with an active eating disorder, who did not differ on these subscales. In contrast, group differences did not emerge for control beliefs or levels of weight/shape self-efficacy. Of note, body shame scores among the non-eating disorder controls and fully recovered group appear somewhat low in this sample (non-eating disorder controls: 2.64; fully recovered group: 2.87), as previous work has indicated means for female college students ranging from 3.23 (Calogero \& Thompson, 2009) to 3.90 (Muehlenkamp \& SarisBaglama, 2002). However, all groups had a mean body surveillance score above the midpoint of this subscale.

\section{Discussion}

The present study sought to examine the components of $\mathrm{OBC}$ across stages of recovery from an eating disorder. Prior research has found associations between body surveillance, body 
shame, and to a lesser degree, beliefs regarding the control one has over the body, and disordered eating (e.g., Striegel-Moore \& Bulik, 2007; Tiggemann \& Kuring, 2004), and to our knowledge, this is the first study to examine these constructs in relation to eating disorder recovery. Results indicated that both body surveillance and body shame differed across stages of recovery from an eating disorder. Individuals who were fully recovered from an eating disorder (i.e., on physical, behavioral, and psychological dimensions) looked like non-eating disorder controls in terms of the degree to which they monitored their bodies and felt shame related to not meeting cultural expectations for the body. In contrast, the partially recovered individuals looked strikingly similar to those with an active eating disorder on body surveillance and body shame. Thus, higher levels of these constructs are associated with a lack of psychological eating disorder recovery (even when physical and behavioral recovery are present) and current eating disorder symptoms.

These results are important in that they highlight the association between low levels of body surveillance and body shame and full eating disorder recovery. Previous research has indeed indicated that eating disorder recovery tends to involve a reconnection with the self (Garrett 1996, 1997), a connection that is arguably disrupted with self-objectification. Eating disorder recovery involves expanding and redefining one's means of self-worth (beyond just body, weight, shape, etc.), and through this process, an individual comes to believe that her body is just a part of who she is rather than all of who she is (Fairburn, 2008). Likewise, lowered levels of body surveillance and body shame among those fully recovered from an eating disorder may indicate that these individuals are learning to reject the notion that bodies should be judged solely on how they look. Further, the development of a more realistic and positive body experience has been posited as an essential step in the eating disorder recovery process (Probst, Van Coppenolle, \& Vandereycken, 1995), and measure development related to eating disorder recovery efficacy has included a subscale assessing the ability to maintain a realistic body image (Pinto, Guarda, Heinberg, \& DiClemente, 2006; Pinto, Heinberg, Coughlin, Fava, and Guarda, 2008), which would be difficult to maintain when high in self-objectification. The results of the present study further support the idea that a reconnection with the self and the body and a rejection of self-objectification characterize full eating disorder recovery.

Interestingly, neither control beliefs, a component of OBC, nor weight/shape self-efficacy differed across recovery group and non-eating disorder controls. Research on these constructs has yielded mixed findings regarding their relations to eating disorder symptomatology (e.g., Cain, Bardone-Cone, Abramson, Vohs, \& Joiner, 2008, 2010; McKinley, 1998, 1999). The current findings add to the body of literature that has not found relations between control beliefs and eating pathology.

This study contributes to the existing literature by being the first to examine the full set of OBC constructs in relation to recovery from an eating disorder, rigorously defined.

Additional strengths include the use of a diagnostic interview, the recruitment through a primary care facility, and the criteria for non-eating disorder controls. By sampling from a facility other than an eating disorders clinic, we were able to examine a broader range of eating disorder severity, contributing to the generalizability of these results. Moreover, by only requiring the absence of a past or current eating disorder in the controls, rather than the absence of any psychopathology, we avoided using a "super healthy" and likely unrepresentative control group (Klump et al., 2004). Finally, by examining results for both the OBCS Control Beliefs subscale and the weight/shape self-efficacy scale across groups, we attempted to clarify previously mixed findings regarding the association between one's belief in the control over the body and eating pathology. 
One limitation of the current study is that we were unable to contact a number of individuals (close to half of the potential study participants) for a variety of reasons, which included last name changes after marriage and cellular phone numbers, which are not recorded in a public registry. While no differences were found between participants and non-participants on relevant eating disorder measures from clinic charts (e.g., diagnoses, BMI at start of treatment), the groups could have differed on unmeasured constructs, which could have introduced bias. Additional limitations include the cross-sectional design, the self-report nature of the questionnaires, the over-representation of Caucasian ethnicity, and the use of non-eating disorder controls that were significantly younger than the rest of our sample, although controlling for age yielded the same pattern of results as without this covariate. Future work should be prospective in nature, so as to examine the temporal ordering of changes in $\mathrm{OBC}$ and eating disorder recovery, and include comparison groups that are agematched. Further, the grouping together of AN, BN, and EDNOS is a limitation, with the current sample size (particularly the small sample sizes of the partial and full eating disorder recovery groups) precluding the separate examination of these diagnostic groups. However, given that migration across eating disorder diagnostic categories is common (Tozzi et al., 2005), examining OBC across combined diagnostic groups may be conceptually appropriate.

Overall, findings of the current study have significant clinical implications. It is likely that changes in body surveillance and body shame precede and influence recovery from an eating disorder. If prospective research bears this out, clinicians would be wise to target these components of self-objectification among individuals with and recovering from eating disorders. For example, feminist theorists have posited that culture defines what is valued in human bodies (e.g., Bordo, 1993), and as such, many feminist models of treatment help individuals develop a critical awareness about societal attitudes toward gender, power, and body shape and size (Faith, Pinhas, Schmelefske, \& Bryden, 2003). Similarly, feminist psychoanalytically informed treatment models strive to aid individuals in renouncing societal judgments about the perfect body and acquiring more flexible standards for the body (Zerbe, 1996). Given OBC's roots in feminist theory, it is believed that using some of the aforementioned treatment approaches might serve to help reduce body surveillance and body shame, which might then aid in recovery. Those who continue to engage in body surveillance and feel shame regarding their body despite an absence of frank eating disorder behaviors may remain partially recovered or relapse back into an active eating disorder. It is acknowledged that some change in body surveillance and body shame may occur after recovery has been achieved and, thus, that self-objectification and the recovery process likely have reciprocal influences.

Results of the present study suggest that individuals who are fully recovered have levels of trait self-objectification similar to those of non-eating disorder controls; yet, future research may wish to examine what happens when a fully recovered individual is put in a selfobjectifying situation (e.g., trying on a one-piece swimsuit; Hebl, King, \& Lin, 2004). Do fully recovered individuals exhibit elevated state self-objectification in comparison to noneating disorder controls? Researchers should also examine the relation between selfobjectification and eating disorder recovery using: a more ethnically diverse group, a longitudinal design that permits capturing the interplay between changes in selfobjectification and eating pathology, and different measures of self-objectification (e.g., Self-Objectification Questionnaire; Fredrickson, Roberts, Noll, Quinn, \& Twenge, 1998; Noll \& Fredrickson, 1998) for comparison purposes. A longitudinal design would also be important in assessing whether levels of body surveillance and body shame among those partially recovered differentiate individuals who are simply on the road to full recovery from individuals who seem to be "stuck" in a more long-lasting partially recovered state. There are also important constructs related to self-objectification, such as thin ideal internalization 
and social comparison, that warrant examination both in terms of the directionality of their relations with self-objectification and how these sets of variables impact eating disorder symptomatology and recovery. For example, Hesse-Biber, Leavy, Quinn, and Zoino (2006) conceptualized objectification theory as an explanation as to why women are particularly susceptible to social comparison (especially in relation to their bodies); yet it seems plausible that social comparison may also predispose women to feel objectified, with selfobjectification and social comparison having a bearing on eating disorder symptoms and the recovery process.

In conclusion, the current study highlights the importance of understanding the relation between body surveillance and body shame and stages of eating disorder recovery. Our results indicate that, for at least some individuals, comprehensive recovery is possible and that this type of recovery is associated with levels of body surveillance and body shame comparable to non-eating disorder controls. Neither levels of control beliefs nor weight/ shape self-efficacy differed across groups, suggesting that beliefs regarding the control one has over the body may be much less closely tied to recovery than the other components of OBC. Overall, this study provides support for the importance of examining objectificationrelated constructs, particularly body surveillance and body shame, across the course of an eating disorder.

\section{Acknowledgments}

Role of Funding Sources This research was supported in part by the following grants to the second author: NIH 1 R03MH074861-01A1; University of Missouri PRIME Grant; and University of Missouri Research Council Grant. Neither NIH nor the University of Missouri had any role in the study design, collection, analysis or interpretation of the data, manuscript preparation, or the decision to submit the paper for publication.

\section{References}

Anderson, DA.; Williamson, DA. Outcome measurement in eating disorders. In: IsHak, WW.; Burt, T.; Sederer, LI., editors. Outcome measurement in psychiatry: A critical review. American Psychiatric Press; Washington, DC: 2002. p. 289-301.

Bardone-Cone, AM. Retrieved from ProQuest Digital Dissertations. 2002. An interactive model of perfectionism, perceived weight status, and self-efficacy in the prediction of bulimic symptoms. (AAI3020840)

Bardone-Cone AM, Cass KM. What does viewing a pro-anorexia website do? An experimental examination of website exposure and moderating effects. International Journal of Eating Disorders. 2007; 40:537-548. doi:10.1002/eat.20396. [PubMed: 17525952]

Bardone-Cone AM, Harney MB, Maldonado CR, Lawson MA, Robinson DP, Smith R, Tosh A. Defining recovery from an eating disorder: Conceptualization, validation, and examination of psychosocial functioning and psychiatric comorbidity. Behaviour Research and Therapy. 2010; 48:194-202. doi:10.1016/j.brat.2009.11.001. [PubMed: 19945094]

Bartky, SL. Femininity and domination: Studies in the phenomenology of oppression. Routledge; New York: 1990.

Basow S, Foran K, Bookwala J. Body objectification, social pressure, and disordered eating behavior in college women: The role of sorority membership. Psychology of Women Quarterly. 2007; 31:394-400. doi:10.1111/j.1471-6402.2007.00388.x.

Bjorntorp, P. Definition and classification of obesity. In: Fairburn, CG.; Brownell, KD., editors. Eating disorders and obesity. 2nd ed. Guilford; New York: 2002. p. 377-381.

Bordo, S. Unbearable weight: Feminist, Western culture, and the body. University of California Press; Berkely, CA: 1993.

Cain AS, Bardone-Cone AM, Abramson LY, Vohs KD, Joiner TE. Refining the relationships of perfectionism, self-efficacy, and stress to dieting and binge eating: Examining the appearance, interpersonal, and academic domains. International Journal of Eating Disorders. 2008; 41:713-721. doi:10.1002/eat.20563. [PubMed: 18537167] 
Cain AS, Bardone-Cone AM, Abramson LY, Vohs KD, Joiner TE. Prospectively predicting dietary restraint: The role of interpersonal self-efficacy, weight/shape self-efficacy, and interpersonal stress. International Journal of Eating Disorders. 2010 doi:10.1002/eat.20740.

Calogero R, Davis W, Thompson J. The role of self-objectification in the experience of women with eating disorders. Sex Roles. 2005; 52:43-50. doi:10.1007/s11199-005-1192-9.

Calogero R, Thompson J. Sexual self-esteem in American and British college women: Relations with self-objectification and eating problems. Sex Roles. 2009; 60:160-173. doi:10.1007/ s11199-008-9517-0.

Couturier J, Lock J. What is recovery in adolescent anorexia nervosa? International Journal of Eating Disorders. 2006; 39:550-555. doi:10.1002/eat.20309. [PubMed: 16791851]

Fairburn, CG., editor. Cognitive behavior therapy and eating disorders. Guilford Press; New York: 2008.

Fairburn CG, Beglin SJ. Assessment of eating disorders: Interview or self-report questionnaire? International Journal of Eating Disorders. 1994; 16:363-370. [PubMed: 7866415]

Faith K, Pinhas L, Schmelefske J, Bryden P. Developing a feminist-informed model for decision making in the treatment of adolescent eating disorders. Eating Disorders. 2003; 11:305-315. doi: 10.1080/10640260390242533. [PubMed: 16864295]

First, MB.; Spitzer, RL.; Gibbon, M.; Williams, JB. Structured Clinical Interview for DSM-IV Axis I Disorders - Patient Edition. Version 2.0. New York State Psychiatric Institute; New York, NY: 1995. SCID-I/P

Fredrickson BL, Roberts T. Objectification theory: Toward understanding women's lived experiences and mental health risks. Psychology of Women Quarterly. 1997; 21:173-206. doi:10.1111/j. 1471-6402.1997.tb00108.x.

Fredrickson BL, Roberts T, Noll SM, Quinn DM, Twenge JM. That swimsuit becomes you: Sex differences in self-objectification, restrained eating, and math performance. Journal of Personality and Social Psychology. 1998; 75:269-284. doi:10.1037/0022-3514.75.1.269. [PubMed: 9686464]

Furnham A, Atkins L. Dieting control beliefs and disordered eating. European Eating Disorders Review. 1997; 5:278-296. doi:10.1002/(SICI)1099-0968(199712)5:4<278::AIDERV171>3.0.CO;2-G.

Garrett C. Recovery from anorexia nervosa: A Durkheimian interpretation. Social Science \& Medicine. 1996; 43:1489-1506. doi:10.1016/0277-9536(96)00088-3. [PubMed: 8923621]

Garrett C. Recovery from anorexia nervosa: A sociological perspective. International Journal of Eating Disorders. 1997; 21:261-272. doi:10.1002/(SICI)1098-108X(199704)21:3<261::AIDEAT6>3.0.CO;2-I. [PubMed: 9097199]

Greenleaf C. Self-objectification among physically active women. Sex Roles. 2005; 52:51-62. doi: 10.1007/s11199-005-1193-8.

Harper B, Tiggemann M. The effect of thin ideal media images on women's self-objectification, mood, and body image. Sex Roles. 2008; 58:649-657. doi:10.1007/s11199-007-9379-x.

Harrison K. Ourselves, our bodies: Thin-ideal media, self-discrepancies, and eating disorder symptomatology in adolescents. Journal of Social and Clinical Psychology. 2001; 20:289-323. doi:10.1521/jscp.20.3.289.22303.

Hebl MR, King EB, Lin J. The swimsuit becomes us all: Ethnicity, gender, and vulnerability to selfobjectification. Personality and Social Psychology Bulletin. 2004; 30:1322-1331. doi: 10.1177/0146167204264052. [PubMed: 15466604]

Herzog DB, Sacks NR, Keller MB, Lavori PW, von Ranson KB, Gray HM. Patterns and predictors of recovery in anorexia nervosa and bulimia nervosa. Journal of the American Academy of Child and Adolescent Psychiatry. 1993; 32:835-842. doi:10.1097/00004583-199307000-00020. [PubMed: 8340307]

Hesse-Biber S, Leavy P, Quinn CE, Zoino J. The mass marketing of disordered eating and eating disorders: The social psychology of women, thinness and culture. Women's Studies International Forum. 2006; 29:208-224. doi:10.1016/j.wsif.2006.03.007.

Klump KL, Strober M, Bulik CM, Thornton L, Johnson C, Devlin B, Kaye WH. Personality characteristics of women before and after recovery from an eating disorder. Psychological Medicine. 2004; 34:1407-1418. doi:10.1017/S0033291704002442. [PubMed: 15724872] 
Laliberte M, Newton M, McCabe R, Mills J. Controlling your weight versus controlling your lifestyle: How beliefs about weight control affect risk for disordered eating, body dissatisfaction and selfesteem. Cognitive Therapy and Research. 2007; 31:853-869. doi:10.1007/s10608-006-9104-z.

Luce KH, Crowther JH. The reliability of the Eating Disorder Examination - Self-report questionnaire version (EDE-Q). International Journal of Eating Disorders. 1999; 25:349-351. doi:10.1002/ (SICI)1098-108X(199904)25:3<349::AID-EAT15>3.0.CO;2-M. [PubMed: 10192002]

McFarland, B.; Baker-Baumann, T. Shame and body image: Culture and the compulsive eater. Health Communications; Deerfield Beach, FL: 1990.

McKinley NM. Gender differences in undergraduates' body esteem: The mediating effect of objectified body consciousness and actual/ideal weight discrepancy. Sex Roles. 1998; 39:113-123. doi:10.1023/A:1018834001203.

McKinley NM. Women and objectified body consciousness: Mothers' and daughters' body experience in cultural, developmental, and familial context. Developmental Psychology. 1999; 35:760-769. doi:10.1037/0012-1649.35.3.760. [PubMed: 10380866]

McKinley NM, Hyde JS. The Objectified Body Consciousness Scale: Development and validation. Psychology of Women Quarterly. 1996; 20:181-215. doi:10.1111/j.1471-6402.1996.tb00467.x.

Mercurio AE, Landry LJ. Self-objectification and well-being: The impact of self-objectification on women's overall sense of self-worth and life satisfaction. Sex Roles. 2008; 58:458-466. doi: 10.1007/s11199-007-9357-3.

Miner-Rubino K, Twenge JM, Fredrickson BL. Trait self-objectification in women: Affective and personality correlates. Journal of Research in Personality. 2002; 36:147-172. doi:10.1006/jrpe. 2001.2343.

Mond JM, Hay PJ, Rodgers B, Owen C. Eating Disorder Examination Questionnaire (EDE-Q): Norms for young adult women. Behaviour Research and Therapy. 2006; 44:53-62. doi:10.1016/j.brat. 2004.12.003. [PubMed: 16301014]

Moradi B. Addressing gender and cultural diversity in body image: Objectification theory as a framework for integrating theories and grounding research. Sex Roles. 2010; 63:138-148. doi: 10.1007/s11199-010-9824-0.

Moradi B. Objectification theory: Areas of promise and refinement. Counseling Psychologist. 2011; 39:153-163. doi:10.1177/0011000010384279.

Muehlenkamp J, Saris-Baglama R. Self-objectification and its psychological outcomes for college women. Psychology of Women Quarterly. 2002; 26:371-379. doi: 10.1111/1471-6402.t01-1-00076.

Myers T, Crowther J. Sociocultural pressures, thin-ideal internalization, self-objectification, and body dissatisfaction: Could feminist beliefs be a moderating factor? Body Image. 2007; 4:296-308. doi: 10.1016/j.bodyim.2007.04.001. [PubMed: 18089276]

Noll SM, Fredrickson BL. A meditational model linking self-objectification, body shame, and disordered eating. Psychology of Women Quarterly. 1998; 22:623-636. doi:10.1111/j. 1471-6402.1998.tb00181.x.

Pinto AM, Guarda AS, Heinberg LJ, DiClemente CC. Development of the Eating Disorder SelfEfficacy Questionnaire. International Journal of Eating Disorders. 2006; 39:376-384. doi:10.1002/ eat.20256. [PubMed: 16528731]

Pinto AM, Heinberg LJ, Coughlin JW, Fava JL, Guarda AS. The Eating Disorder Recovery SelfEfficacy Questionnaire (EDRSQ): Change with treatment and prediction of outcome. Eating Behaviors. 2008; 9:143-153. doi:10.1016/j.eatbeh.2007.07.001. [PubMed: 18329592]

Probst M, Van Coppenolle H, Vandereycken W. Body experience in anorexia nervosa patients: An overview of therapeutic approaches. Eating Disorders. 1995; 3:145-157. doi: 10.1080/10640269508249157.

Reas DL, Williamson DA, Martin CK, Zucker NL. Duration of illness predicts outcome for bulimia nervosa: A long-term follow-up study. International Journal of Eating Disorders. 2000; 27:428434. doi:10.1002/(SICI)1098-108X(200005)27:4<428::AID-EAT7>3.0.CO;2-Y. [PubMed: 10744849]

Segal DL, Hersen M, Van Hasselt VB. Reliabililty of the Structured Clinical Interview for DSM-III-R: An evaluative review. Comprehensive Psychiatry. 1994; 35:316-327. [PubMed: 7956189] 
Shear MK, Greeno C, Kang J, Ludewig D, Frank E, Swartz HA, Hanekamp M. Diagnosis of nonpsychotic patients in community clinics. American Journal of Psychiatry. 2000; 157:581-587. doi:10.1176/appi.ajp.157.4.581. [PubMed: 10739417]

Sherer M, Adams CH. Construct validation of the Self-Efficacy Scale. Psychological Reports. 1983; 53:889-902.

Sherer M, Maddux JE, Mercandante B, Prentice-Dunn S, Jacobs B, Rogers RW. The Self-Efficacy Scale: Construction and Validation. Psychological Reports. 1982; 51:663-671.

Smith GT, Hohlstein LA, Atlas JG. Accuracy of self-reported weight: Variation with binger or restrainer status and eating disorder symptomatology. Addictive Behaviors. 1992; 17:1-8. doi: 10.1016/0306-4603(92)90047-Y. [PubMed: 1595421]

Steiner JL, Tebes JK, Sledge WH, Walker ML. A comparison of the Structured Clinical Interview for DSM-III-R and clinical diagnoses. Journal of Nervous and Mental Disease. 1995; 183:365-369. doi:10.1097/00005053-199506000-00003. [PubMed: 7798084]

Striegel-Moore RH, Bulik CM. Risk factors for eating disorders. American Psychologist. 2007; 62:181-198. doi:10.1037/0003-066X.62.3.181. [PubMed: 17469897]

Tiggemann M, Kuring JK. The role of body objectification in disordered eating and depressed mood. British Journal of Clinical Psychology. 2004; 43:299-311. doi:10.1348/0144665031752925. [PubMed: 15333234]

Tiggemann M, Slater A. A test of objectification theory in former dancers and non-dancers. Psychology of Women Quarterly. 2001; 25:57-64. doi:10.1111/1471-6402.00007.

Tozzi F, Thornton LM, Klump KL, Fichter MM, Halmi KA, Kaplan AS, Kaye WH. Symptom fluctuation in eating disorders: Correlates of diagnostic crossover. American Journal of Psychiatry. 2005; 162:732-740. doi:10.1176/appi.ajp.162.4.732. [PubMed: 15800146]

Tylka T. The relation between body dissatisfaction and eating disorder symptomatology: An analysis of moderating variables. Journal of Counseling Psychology. 2004; 51:178-191. doi: 10.1037/0022-0167.51.2.178.

Tylka TL, Hill MS. Objectification theory as it relates to disordered eating among college women. Sex Roles. 2004; 51:719-730. doi:10.1007/s11199-004-0721-2.

Yackobovitch-Gavan M, Golan M, Valevski A, Kreitler S, Bachar E, Lieblich A, Stein D. An integrative quantitative model of factors influencing the course of anorexia nervosa over time. International Journal of Eating Disorders. 2009; 42:306-317. doi:10.1002/eat.20624. [PubMed: 19040269]

Zanarini MC, Skodol AE, Bender D, Dolan R, Sanislow C, Schaefer E, Gunderson JG. The collaborative longitudinal personality disorders study: Reliability of axis I diagnoses. Journal of Personality Disorders. 2000; 14:291-299. [PubMed: 11213787]

Zerbe KJ. Feminist psychodynamic psychotherapy of eating disorders: Theoretic integration informing clinical practice. Psychiatric Clinics of North America. 1996; 19:811-827. doi:10.1016/ S0193-953X(05)70383-X. [PubMed: 8933610] 


\section{Research Highlights}

- Controls and fully recovered women had equally low levels of body surveillance and body shame

- Partially recovered women looked like eating disorder cases on these constructs

- Control beliefs and weight/shape self-efficacy did not differ across groups

- Examining self-objectification in relation to eating disorder recovery is important 


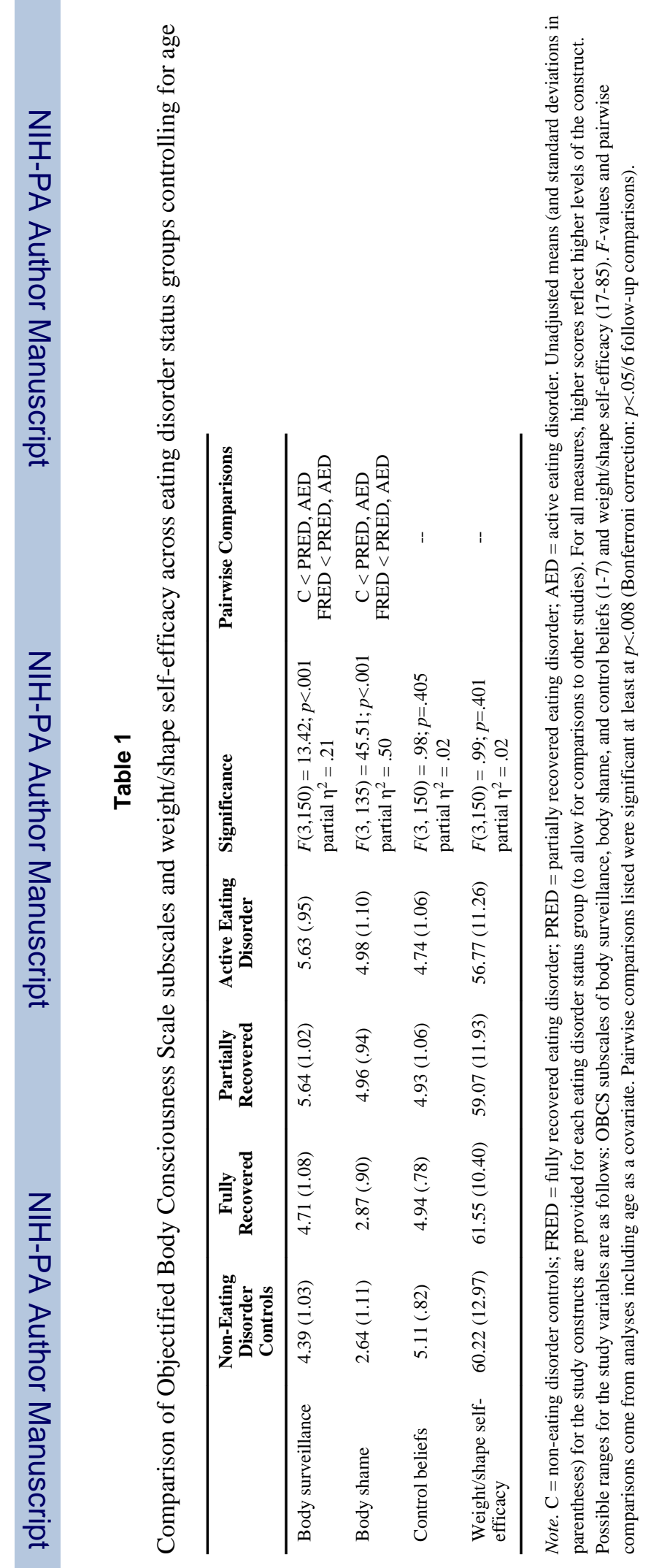

Eat Behav. Author manuscript; available in PMC 2012 December 1. 